IT keineswegs einheitlich beurteilt. Das Absetzen vor einer IT mit (allen!) Allergenen ist m.E. bis jetzt nicht gut begründet. Nicht nachvollziehen kann ich, dass sie auch vor Hauttestungen, einschließlich Pricktestungen, nicht verabreicht werden sollten.

\section{Abklärungsbedarf vorhanden}

Wie man mit den Hauttests bei einer laufenden Behandlung von Testpatienten mit ACE-Hemmern und Betablockern umgehen sollte, müsste von den zuständigen Gremien des Ärzteverbandes Deutscher Allergologen und der Deutschen Gesellschaft für Allergologie und klinische Immunologie zusammen mit dem Paul-EhrlichInstitut erarbeitet werden. Für die Praxis erscheint das nicht zuletzt aus juristischen Gründen notwendig.

\section{Literatur}

1. Malling H-J, ed. Immunotherapy. Position Paper. Allergy 1988; 43: Suppl. 6.

2. Leonhardt L, Kersten W. Empfehlung zur „Immuntherapie“" (spezifische Hyposensibilisierung). Allergologie 1988; 11: 518.

3. Kaplan AP, et al. Betaadrenergic blockers, immunotherapy, and skin testing. J Allergy Clin Immunol 1989; 84:129.

4. Dreborg S, ed. Skin tests used in type I allergy testing. Position paper EAACI. Allergy 1989; 44: Suppl. 10.

5. Bousquet J, Lockey RF, Malling H-J, ed. WHO Position Paper Allergen immunotherapy: therapeutic vaccines for allergic diseases. Allergy 1998; 53: Suppl.

6. Bergmann KC, Müsken H. Durchführung und Bewertung des Pricktests. Allergo J 1992; 1: 56.

7. Bergmann KC, Müsken H. Durchführung und Bewertung des Intrakutantests. Allergo J 1992; 2: 55.

8. Bemstein IL, Storms WW. EDS. Practice parameters for allergy diagnostic testing. Ann Allergy 1995; 75: 56 .
9. Kleinhans D. Allergische Nebenwirkungen bei Hauttestungungen. Vortrag 21. Tagung der Dtsch. Ges. für Allergologie und klin. Immunologie. München 1999.

10. Kleinhans D. Anaphylaktoide Reaktionen bei Patienten unter ACE-Hemmer-Therapie bei gleichzeitiger Desensibilisierungsbehandlung oder nach Insektenstichen. Allergo J 1995; 4: 296.

11. Arzneimittelkommission der Deutschen Ärzteschaft: Anaphylaktoide Reaktionen bei Patienten mit ACE-Hemmer-Behandlung bei gleichzeitiger Desensibilisierungsbehandlung oder nach Insektenstichen. Dtsch Ärztebl 1994; 91: B 1762.

12. Lüderitz-Püchel U. Persönliche Mitteilung.

13. Sennekamp J, Kersten W, Hornung B. Empfehlung zur Hyposensibilisierung mit Allergenextrakten. Allergo J 1995; 4: 205.

\section{Prof. Dr. med. Dieter Kleinhans}

Kolpingstrasse 2, 70736 Fellbach

\title{
Berufspolitik
}

\section{EBM-Laborreform - neuer Maßnahmenkatalog}

\section{Am 16. Februar dieses Jahres wurde von dem Bewertungsausschuss der Ärzte und Krankenkassen ein Katalog von Maßnahmen beschlossen, der die EBM-Laborreform betrifft.}

Vor allem folgende Punkte sind für allergologisch tätige Ärzte interessant:

> Auf alle Kostensätze bei EBMZiffern des Kapitels OIII gibt es seit dem 1. Januar 2000 bis vorläufig zum 31. Dezember 2000 einen Aufschlag von 24\%.

- Dieser Zuschlag gilt nur für solche Laboratorien, die weniger als 450000 Analysen pro Quartal durchführen.

- In den nächsten sechs bis neun Monaten werden alle EBM-O-IIIKostensätze überprüft und neu festgesetzt.
> Neu aufgenommen werden u.a. in den Ausnahmekatalog GOP 3484 allergische Erkrankungen bei Kindern bis zum sechsten Lebensjahr.

Der Maßnahmenkatalog enthält weitere Änderungen insbesondere bezüglich der mikrobiellen Diagnostik. Hierauf wird nicht näher eingegangen.

Die Maßnahme dient dazu, die Auswirkung der Laborreform zu mildern. Dies wird vor allem den allergologisch tätigen Kollegen mit einem Einsendelabor zugute kommen.

Dr. med. Werner Kersten

\section{IGeL-Liste erweitert}

Dr. med. Lothar Krimmel hat die IGeL-Liste erweitert und die Institution „Medwell“ gegründet. Medwell informiert interessierte Ärzte über alle IGeL-Leistungen.

Telefonisch ist das Institut unter der Nummer 0 1805/468-468 zu erreichen, die Briefadresse lautet: Medwell, Im Winkel 1-5, 51145 Köln.

Unter www.medwell.de sind weitere Informationen erhältlich. So besteht auch die Möglichkeit, die erweiterte IGeL-Liste auf den heimischen PC herunter zu laden.

Teilnehmer des diesjährigen ÄDAKongresses am 3. September in Bonn (s. Anzeige in diesem Heft) können Dr. Krimmel auch live hören und mit ihm diskutieren.

Weitere berufspolitische Aspekte werden auf dieser Veranstaltung in der anschließenden Podiumsdiskussion behandelt.

Prof. Dr. med. Joachim Sennekamp 